\title{
Sample size calculation for clinical trials: the impact of clinician beliefs
}

\author{
PM Fayers ${ }^{1}$, A Cuschieri ${ }^{2}$, J Fielding ${ }^{3}$, J Craven $^{4}$, B Uscinska ${ }^{1}$ and LS Freedman ${ }^{5}$ \\ ${ }^{1}$ Cancer Division, MRC Clinical Trials Unit, 222 Euston Road, London NW1 2DA, UK; ${ }^{2}$ University Department of Surgery, Ninewells Hospital and Medical \\ School, Dundee DD1 9SY, UK; ${ }^{3}$ Department of Surgery, Queen Elizabeth Hospital, Birmingham B15 2TH, UK; ${ }^{4}$ Kingstown General Hospital, St Vincents, \\ West Indies; ${ }^{5}$ Department of Mathematics, Statistics and Computer Science, Bar Ilan University, Ramat Gan 52900, Israel
}

\begin{abstract}
Summary The UK Medical Research Council (MRC) randomized trial of gastric surgery, ST01, compared conventional (D1) with radical (D2) surgery. Sample size estimation was based upon the consensus opinion of the surgical members of the design team, which suggested that a change in 5 -year survival from $20 \%$ (D1) to $34 \%$ (D2) could be realistic and medically important. On the basis of these survival rates, the sample size for the trial was 400 patients. However, this trial was exceptional in the way that a survey of surgeons' opinions was made at the start of the trial, in 1986, and again before results were analysed but after termination of the trial in 1994. At the initial survey, the three surgeons from the trial steering committee and 23 other surgeons experienced in treating gastric carcinoma were given detailed questionnaires. They were asked about the expected survival rate in the D1 group, anticipated difference in survival from D2 surgery, and what difference would be medically important and influence future treatment of patients. The consensus opinion of those surveyed was that there might be a survival improvement of $9.4 \%$. In 1994, prior to closure of the trial, and before any survival information was disclosed, the survey was repeated with 21 of the original 26 surgeons. At this second survey, the opinion of the trial steering committee was that $9.5 \%$ difference was more realistic. This was in accord with the opinion of the larger group, which remained little changed since 1986 . The baseline 5-year D1 survival was thought likely to be about $32 \%$, which corresponded closely to the actual survival of recruited patients. Revised sample size calculations suggested that, on the basis of these more recent opinions, between 800 and 1200 patients would have been required. Both surveys assessed the level of treatment benefit that was deemed to be sufficient for causing surgeons to change their practice. This showed that the $13 \%$ difference in survival used as the study target was clinically relevant, but also indicated that many clinicians would remain unwilling to change their practice if the difference is only $9.5 \%$. The experience of this carefully designed trial illustrates the problems of designing long-term, randomized trials. It raises interesting questions about the common practice of basing sample size estimates upon the beliefs of a trial design committee that may include a number of enthusiasts for the trial treatment. If their opinion of anticipated effect sizes drives the design of the trial, rather than the opinion of a larger community of experts that includes sceptics as well as enthusiasts, there is likely to be a serious miscalculation of sample size requirements. (C) 2000 Cancer Research Campaign
\end{abstract}

Keywords: sample size; prior beliefs; clinicians' opinions; clinical trials

In 1986 the UK Medical Research Council (MRC) designed a randomized clinical trial (ST01) comparing D1 versus D2 surgery for operable gastric cancer. At that time D1 surgery was the conventional surgical procedure for gastric surgery in the West, while D2 radical surgery with extended lymph node dissection was standard practice in Japan. Japanese reports, based upon nonrandomized studies, indicated probable major survival benefits associated with D2 surgery. Five-year survival of (Japanese) D2 patients was nearly double that of (Western) D1 patients (Maruyama et al, 1987). However, it was unknown whether these results were partly or entirely due to factors such as patient selection and rigorous staging classification. The MRC trial was designed to address these questions, using randomization to ensure an unbiased comparison. The principal end point of the study was 5 -year survival. One noteworthy aspect of this trial is that it represents one of the earliest examples of a purely surgical randomized

Received 28 October 1998

Revised 22 February 1999

Accepted 8 July 1999

Correspondence to: PM Fayers. E-mail: PFayers@ hotmail.com trial in cancer, and to date there are still very few trials comparing two or more alternative methods of surgery.

\section{METHODS}

\section{Design of ST01 trial}

The ST01 trial design team, which later formed the trial steering committee, included three consultant surgeons with an interest in treatment of gastric cancer. Patients with resectable advanced gastric cancer were to be randomized between the standard UK operation (variations on the D1 theme) and the more radical D2 resection practised by members of the Japanese Research Society for Gastric Cancer. Eligible patients were defined as potentially curative $\mathrm{S}_{0-2} \mathrm{P}_{0} \mathrm{H}_{0} \mathrm{~N}_{0-2}$, that is stage I-III gastric cancer, without positive infracolic aortic nodes. All patients underwent staging laparotomy to confirm potentially curative disease. Eligible consenting patients fit for either D1 or D2 surgery were then randomized centrally (over the phone) within the same operating session. More extensive details of the staging and surgical procedures have been published elsewhere (Cuschieri et al, 1996, 1999).

When the trial was being designed in 1985-86, there was considerable uncertainty about the range of plausible and 


\section{A SURVEY OF SURGEONS' OPINIONS IN RELATION TO THE MRC GASTRIC CANCER SURGERY TRIAL.}

A: All of you will have had considerable experience with DI surgery but most will have been using D2 resection for nearly the first time in this trial. You may possibly feel that the resection is more difficult, hazardous for the patient, time consuming or inconvenient than DI resection. Your opinion may have been altered by your continued experience with D2 resection as the trial progressed.

Primarily we are interested in how seriously you rate the possible extra difficulty of the $D 2$ resection by asking you to balance this against a proposed improvement in the 5 -year survival rate from this operation. Suppose you have been told on good authority the exact improvement you would obtain by treating patients with D2 resection. If there was exactly zero improvement you would presumably use DI resection in the future. If there was a $30 \%$ improvement (ie from $25 \%$ up to $55 \% 5$. year survival) you would presumably use $\mathrm{D} 2$. Somewhere in between these figures there is likely to be a difference where you would change from D1 to D2. There may be a range of differences where the decision would not be clearcut ie a range where you felt the two operations to be roughly equivalent. Please mark the changeove point or this range on the scale of treatment differences shown below. In making your decision you will be weighing the possible extra difficulty of $D 2$ with a given improvement in survival rate.
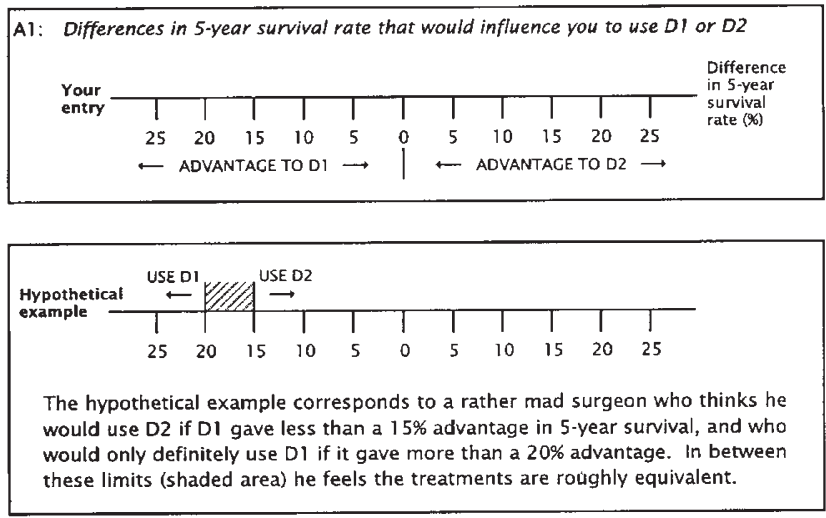

PLEASE FILL IN YOUR OWN JUDGEMENT ON THE SCALE ABOVE THE EXAMPLE

Figure 1 Extract from the survey questionnaire

clinically important survival benefits that might follow D2 surgery. However, this information was necessary for the estimation of sample size (Fayers and Machin, 1995). From past experience in UK, the baseline survival at 5 years for such patients undergoing a D1 resection was judged to be $20 \%$. This estimate was obtained as a consensus opinion of the three surgical members of the ST01 design team. Japanese figures, based upon observational (non-randomized) studies suggested a large survival advantage to D2 surgery, but this could have been influenced by other factors. These included (a) earlier diagnosis, through screening and public awareness; (b) stage migration, since the extensive surgery enabled more rigorous classification and detection of poor-prognosis patients; and (c) physical fitness or other characteristics of the patients, since Japanese patients tend to be less obese and younger than Western patients. Thus members of the ST01 design team had varying opinions as to the magnitude of any survival advantage in favour of D2. The ST01 team was also aware that D2 surgery, being far more extensive than D1, was likely to be accompanied by increased post-operative morbidity and mortality. Thus there would have to be reasonably large long-term survival advantages for D2 to become the treatment of choice. For sample-size calculations we therefore assumed the 5-year survival would be $20 \%$, and that the target treatment difference would be about $13 \%$.

The protocol specified that the main analysis would be based upon a comparison of survival using the log-rank test. With a baseline survival rate of $20 \%, 400$ patients (200 per treatment arm)
C: We are also interested in your expectations of the difference in 5-year survival rates which might result from employing D2 rather than D1 resection for eligible patients. We are not asking you to predict the results of this individual trial but rather the result which would eventually emerge from experience with many thousands of patients. Naturally you are not sure what difference would really emerge and you are likely to feel that differences over a wide range are plausible. Nevertheless you many feel that there are some parts of this range which are more plausible than others. We therefore ask you to enter your weight of belief in each of the possible intervals of difference shown in the table below. The stronger you believe that the difference will truly lie in a given interval the greater should be your weight for that interval. If you believe it impossible that the differences lie in a given interval your weight should be zero. Your weights should add up to 100 .

(NB: By $0-5 \%$ advantage to $D 2$ we mean that the 5 -year survival rate for $D 2$ will be between $0 \%$ and $5 \%$ more than that for D1 resection; eg if 5 -year rate for D1 is $25 \%$ it will be $25-30 \%$ for D2.)

$\mathrm{Cl}$ : Expected differences in 5-year survival rates for $D 1$ versus $D 2$ 5-year survival rate

\begin{tabular}{|l|c|c|c|c|c|c|c|c|c|c|c|c|c|}
\hline \multicolumn{4}{|c|}{ D2 worse than D1 by (\%) } & \multicolumn{6}{c|}{ D2 better than D1 by (\%) } \\
\hline \begin{tabular}{|l|l|l|l|l|l|l|l|l|l|}
\hline Your \\
entry:
\end{tabular} & $25+$ & $20-25$ & $15-20$ & $10-15$ & $5-10$ & $0-5$ & $0-5$ & $5-10$ & $10-15$ & $15-20$ & $20-25$ & $25+$ & Total \\
\cline { 2 - 11 } & & & & & & & & & & & & 100 \\
\hline
\end{tabular}

\begin{tabular}{|c|c|c|c|c|c|c|c|c|c|c|c|c|c|}
\hline \multirow[b]{3}{*}{$\begin{array}{l}\text { Hypothetical } \\
\text { example: }\end{array}$} & \multicolumn{6}{|c|}{ D2 worse than D1 by (\%) } & \multicolumn{7}{|c|}{ D2 better than D1 by (\%) } \\
\hline & $25+$ & $20-25$ & $15-20$ & $10-15$ & $5-10$ & $0-5$ & $0-5$ & $5-10$ & $10-15$ & $15-20$ & $20-25$ & $25+$ & Total \\
\hline & 20 & 20 & 0 & 0 & 0 & 0 & 20 & 0 & 0 & 20 & 0 & 20 & 100 \\
\hline $\begin{array}{l}\text { The hy } \\
\text { equally } \\
25 \% \text { or } \\
25+\%\end{array}$ & $\mathrm{~b} 2$ is & $\begin{array}{l}\text { ette } \\
\text { to ot }\end{array}$ & than & $\begin{array}{l}\text { le cor } \\
\text { worse } \\
\text { D1 by } \\
\text { feren }\end{array}$ & $\begin{array}{l}0-5 \% \\
\text { ces at }\end{array}$ & $\begin{array}{l}\text { ads } t \\
\mathrm{D}^{2} \text { by } \\
\text { or } \mathrm{D2} \\
\text { e imp }\end{array}$ & be & $\begin{array}{l}\text { her } \\
\text { \% or } \\
\text { ter b }\end{array}$ & $\begin{array}{l}\text { nad } 5 \\
2 \text { is } \\
15-2\end{array}$ & $\begin{array}{l}\text { urgeo } \\
\text { Norse } \\
\text { O\% or }\end{array}$ & $\begin{array}{l}n \text { who } \\
\text { than } \\
\text { D2 is }\end{array}$ & $\begin{array}{l}\text { beli } \\
1 \text { by } \\
\text { eette }\end{array}$ & $\begin{array}{l}\text { ves } \\
20 \text { - } \\
\text { by }\end{array}$ \\
\hline
\end{tabular}

PLEASE FILL IN YOUR OWN BELIEF IN THE ROW OF THE TABLE ABOVE THE EXAMPLE

would enable an improvement of $13.5 \%$ (to $33.5 \%$ ) to be detected with a 5\% $P$-value and $90 \%$ power (Machin et al, 1997). Therefore, in 1986 the ST01 trial was launched with a target sample size of 400 randomized patients. Since the eventual analysis would depend upon comparison of survival rates, it would only be possible to analyse the data after sufficient 'events', in this case deaths, had occurred (Fayers and Machin, 1995). The protocol specified that detailed analysis would be deferred until after the accumulation of 250 deaths in the trial.

\section{First survey of surgeons' opinions}

The sample size estimation depends crucially upon the magnitude of the anticipated treatment effect. Whilst 400 patients suffice to be reasonably confident ( $90 \%$ power) of detecting a $13 \%$ improvement, one would need nearly 700 patients for a $10 \%$ improvement. On the other hand, only about 200 patients are needed for detection of a $20 \%$ improvement. The baseline survival rate $(20 \%$ 5-year survival in D1 patients) is less critical to the sample size estimation, but it is particularly important to be confident about the magnitude of the treatment effect that one hopes to detect. The expectations regarding the benefit of D2 surgery were investigated through a survey of surgeons.

Prior to launching the trial, eight surgeons experienced in gastric surgery, including the three surgical members of the steering committee, were individually interviewed, and another 18 intending trial participants completed a postal questionnaire. The 
survey asked 'what differences in 5-year survival rate would influence you to use D1 or D2?' This emphasizes one often overlooked aspect of clinical trials, namely that the role of a clinical trial should not be merely to establish treatment differences, but should be to influence medical practice. The surgeons knew that D2 surgery was more extensive, carried extra risk of complications, and demanded extra resources. If D2 surgery offers no survival benefit, D1 should be the operation of choice. If D2 offers substantial survival benefit, it should be the treatment of choice in suitable patients. But there may be a range of small survival benefit within which surgeons remain uncertain as to whether D1 or D2 is appropriate. This is called the 'range of equivalence'. Therefore, surgeons could specify a range of values within which they would remain uncertain whether to use D1 or D2, and if the results of the trial suggested that the survival difference between D1 and D2 lay within this range the surgeon would have no strong preference for either form of surgery. This approach probably reflects clinical thinking more closely than if one demanded a single value for the treatment difference, above which treatment 1 is preferred and below which treatment 2 is favoured. A clear decision can only be made if the survival difference is found to lie outside the range of equivalence. If the results are more extreme in favour of D2 surgery, the surgeon would prefer D2. Similarly, if results are outside the range of equivalence in the opposite direction, they would choose D1.

A clinical trial should also be realistic. It would be of little relevance to design and conduct a clinical trial on the basis of seeking larger survival benefits than can reasonably be expected to be present. Therefore, the surgeons were asked to indicate what difference in survival they expected would emerge if many patients were given the two treatments. They had to indicate a range of values, and weight their beliefs. For example, a surgeon could have indicated an expected 5-year survival advantage to D2 surgery of $20 \%$ or more. The surgeon could then indicate with decreasing confidence that it could be above $25 \%$, or even $30 \%$ or more. In this manner a 'prior distribution' of beliefs for each surgeon was constructed.

The survey also included various other questions concerning the expected baseline 5-year survival for D1, their level of experience with D2 surgery, and factors influencing their answers (such as the perceived risk of post-operative complications associated with D2). Figure 1 shows extracts from the questionnaire used in the survey.

\section{Second survey of surgeons' opinions}

Before the trial was completed, and before any results had been revealed, a second survey was carried out. The same surgeons, where traceable, were approached. They were asked to complete the same questionnaire as 8 years previously. This time, however, they were additionally asked whether they thought their opinions regarding the expected benefits of D2 had changed over the intervening 8 years.

\section{Impact of ST01 results upon clinical opinion}

The purpose of a clinical trial is to influence clinical opinion when treating similar patients in future. If the results of ST01 show a survival advantage to D2 surgery that is both statistically and clinically significant, those surgeons with pre-study 'prior' beliefs that D2 is superior would presumably become more strongly convinced of its efficacy whilst those who were sceptical regarding D2 surgery would be less strongly swayed in its favour. Statistical methods for combining prior beliefs with the observed data from a clinical trial have been described by Fayers et al (1997). These 'Bayesian' methods enable an estimation of the revised, or 'posterior', beliefs that we would expect the surveyed surgeons to hold after they are told the trial results.

\section{RESULTS}

\section{Pre-study survey, 1986}

\section{Baseline survival rate for D1 patients}

The average estimated value of the baseline 5-year survival for D1 patients was $21 \%$ by the three surgeons on the trial steering committee. This agrees with the overall estimate by the total 26 participating surgeons surveyed, which was 18\% (95\% confidence interval (CI) 15-22). It confirmed the initial informal pre-study estimate of $20 \%$ for 5 -year survival as a realistic assumption for sample-size calculations.

\section{Clinically worthwhile difference}

Most clinicians indicated that a 5-10\% 5-year survival advantage to D2 would leave them uncertain whether to use D1 or D2 surgery; some chose $10-15 \%$ as equivalent, whilst a few chose lower limit of 0 or an upper limit of $20 \%$. The average overall 'range of equivalence' was $4.6-10.8 \%$. The average range of equivalence for the three surgeons of the trial steering committee was $2-7 \%$, suggesting that the steering committee were more willing than other surgeons to accept a small survival benefit as indicating that D2 was worthwhile. That is, they were enthusiasts for the D2 treatment.

\section{Expected difference between D1 and D2}

Figure 2 shows the opinions of the 26 surgeons as to the likely outcome of the clinical trial. The mean value for the three surgical members of the steering committee is $13.1 \%$. This is also above the range of equivalence, and therefore represents a belief that D2 surgery offers a realistic and worthwhile survival benefit. However, the larger group of surgeons was more cautious and on average thought the survival advantage to D2 was more likely to be $9.4 \%$. There was considerable variation in the opinions expressed, and the $95 \%$ CI for the mean expected survival difference was $5.6-11.0 \%$. This represents a feeling that the results of the trial would probably indicate a barely worthwhile advantage to D2, since $9.4 \%$ is at the upper limit of most surgeons' range of equivalence.

\section{Implication for sample-size estimation}

The values entered on the questionnaire by the trial steering committee were consistent with the opinions that they had previously expressed and which had been used for the sample-size estimation in ST01. The larger sample of surgeons were rather more sceptical about expected benefits of D2, and a sample size nearly twice as large would have been necessary if $9.4 \%$ were selected as the target improvement in survival. More details of this technique of assessing clinicians' opinions, together with a general review of sample-size estimation, is available in Fayers and Machin (1995). 


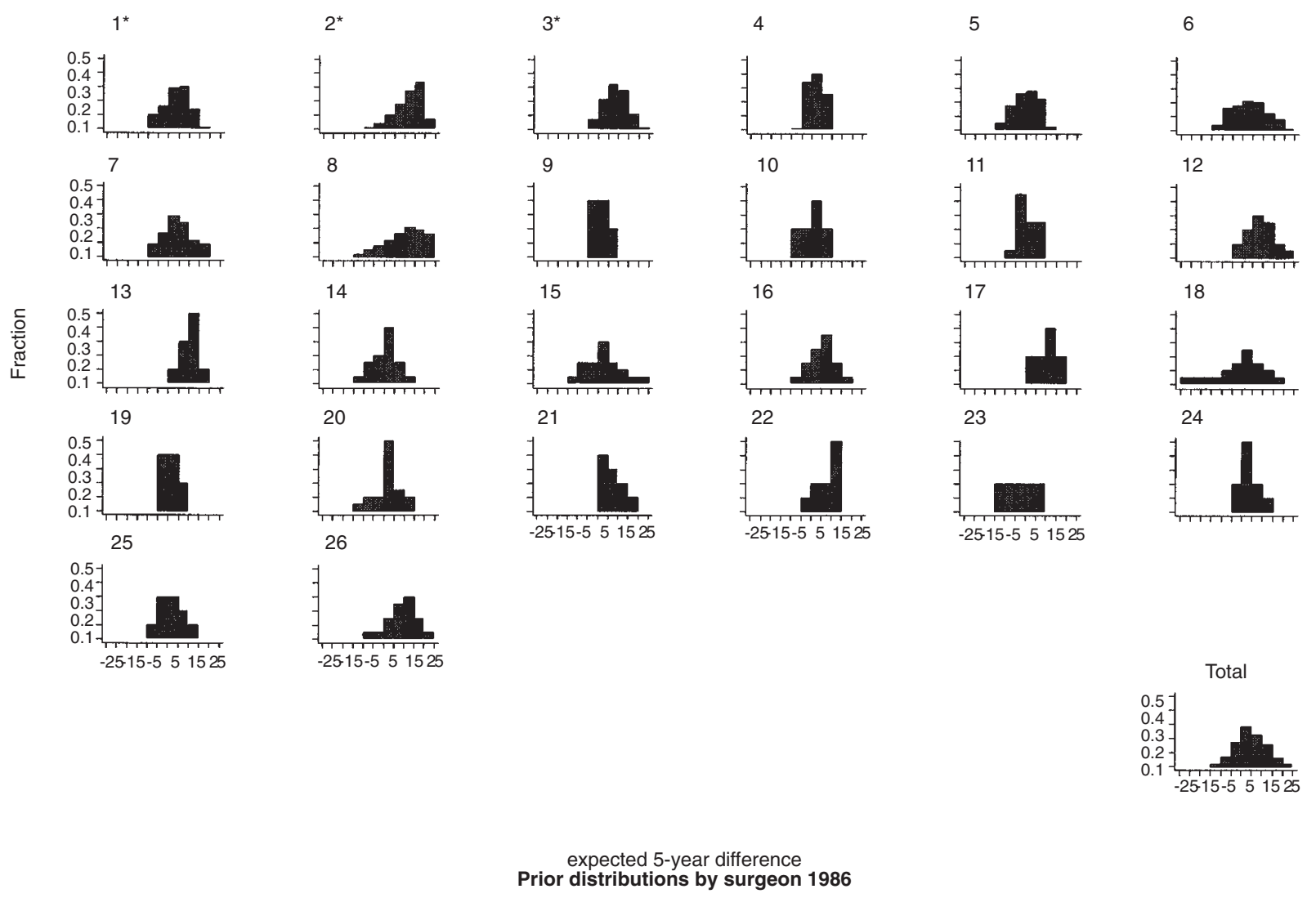

Figure 2 Expected percentage difference in 5-year survival between D1 and D2 surgery, as predicted by 26 experienced surgeons in 1986 . The overall average results are shown to the bottom, right. Steering committee members (surgeons $1-3$ ) are indicated*. Mean expected difference $9.4 \%$ better survival in D2 patients, 95\% confidence interval $5.6-11.0 \%$

\section{Second survey, 1994}

Twenty-one of the original 26 surgeons, including the three steering committee members, were traced and agreed to complete the survey in 1994. Of the other five, one had died, one refused, one could not be traced, and two had retired and did not feel able to pass an opinion.

\section{Baseline survival rate for $D 1$ patients}

The trial steering committee surgeons now thought that the baseline survival for D1 was likely to be $32 \%$ at 5 -years - an increase of $10 \%$ over the initial beliefs 8 years earlier. However, when all 26 surgeons were included, the results showed no statistically significant overall change of opinion (18\% in 1986, $21 \%$ in 1994).

\section{Clinically worthwhile difference}

There was hardly any change in the overall range of equivalence, which now ranged from $5.4 \%$ to $10.6 \%$. The revised range for the steering committee was now $6.0-10.7 \%$, closely similar to that of all surveyed surgeons.

\section{Expected difference between $D 1$ and $D 2$}

There was a small shift in the opinions of the 21 surgeons in the later survey, from the 1986 average of $9.4 \%$ to $8.3 \%$ at the end of the study $(95 \%$ CI $7.8-11.1 \%)$. The overall results are shown in Figure 3. However, the initial enthusiasm of the steering committee surgeons had decreased substantially - from $13.1 \%$ originally, to $9.5 \%$ at the end of the study. Thus the revised opinions of the steering committee are within the bounds of the confidence interval from the total surveyed group.

\section{Sample-size estimation}

Suppose we were designing the ST01 trial in 1995. The expected benefit conferred by D2 would have been estimated as $9.5 \%$. Using a baseline of $20 \% 5$-year survival, about 750 patients would be necessary. However, the baseline survival rate would have been estimated at $32 \%$, which is substantially greater than the originally estimated $20 \%$. Calculations show that the power to detect an improvement of $9.5 \%$, from $32 \%$ 5-year survival for D1 to $41.5 \%$ for D2, would have fallen to $54 \%$ if the study size were specified as 400 patients. Such a low power is unacceptable. To maintain $90 \%$ power, the recruitment would have had to be extended to 1000 patients.

\section{Results of the trial}

In 1993 the ST01 gastric cancer trial completed its intended patient accrual of 400 patients. Analysis of post-operative mortality and morbidity confirmed that, as anticipated, there was increased post-operative mortality associated with D2 surgery, with $6.5 \%$ of D1 and $13 \%$ of D2 patients dying within 30 days or without leaving the hospital (Cuschieri et al, 1996). These results were closely similar to the findings of a Dutch randomized trial comparing the same two operations, in which post-operative mortality rates in patients allocated to D1 and D2 surgery were $6 \%$ and $10 \%$. 


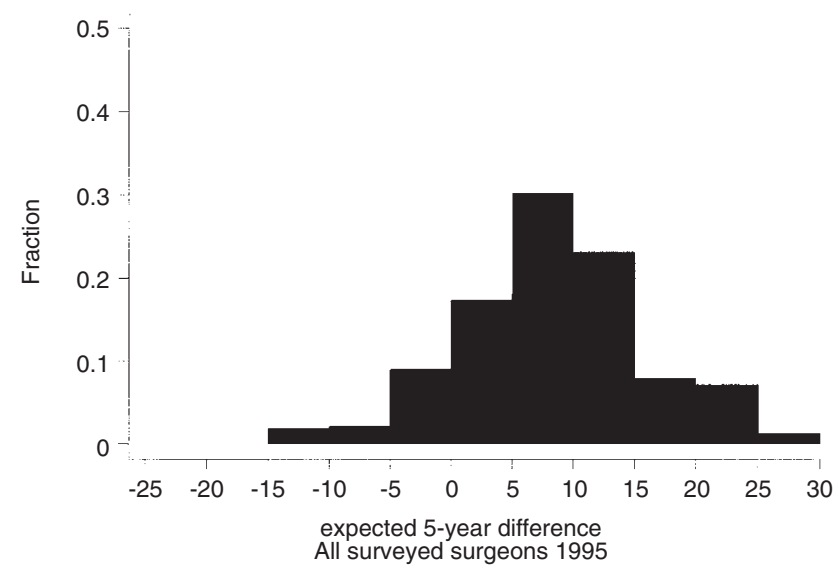

Figure 3 Expected percentage difference in 5-year survival between D1 and D2 surgery, as predicted by 21 experienced surgeons in 1995. Mean expected difference: $8.3 \%$ better survival in D2 patients, $95 \%$ confidence interval 7.8-11.1

The 5-year survival rates have now been published (Cuschieri et al, 1999). The overall patient survival at 5 years was $34 \%(95 \%$ CI $29-39 \%$ ). The two treatment arms were very similar, and D2 surgery did not appear to offer any significant benefit over D1 (hazard ratio $=1.10,95 \%$ CI $0.87-1.30$ ). Figure 4 shows the survival curves, and the impact of the initial post-operative mortality seems to be largely sustained throughout the curves.

The ST01 trial 5-year survival within the D1 group was 30\%, which was closely similar to the baseline survival of $32 \%$ that was anticipated in 1994 (second survey) by the steering committee. It contrasts markedly with the $20 \%$ that was predicted by both the steering committee in 1986 and by the other surgeons in 1986 and 1994.

\section{Estimate of impact of ST01 results}

The observed results can be adjusted to allow for the strong belief that many surgeons have concerning survival advantages due to D2 surgery. The technical details of this 'Bayesian approach' are described, with simple worked examples, in the tutorial by Fayers et al (1997) and in Parmar et al (1994). Briefly, the survival probabilities for the two treatment groups are converted into log hazard ratios, because these are statistically more convenient for analysis. A hazard ratio of greater than 1 indicates an advantage to D1 (as found in the ST01 results), and this corresponds to a log hazard of greater than zero. The prior opinions shown in Figure 3, combined with the mean baseline estimate for the D1 survival rate $(21 \%)$, have a mean log hazard ratio of 0.232 and a standard deviation of 0.226. Applying the Bayesian methodology, we obtain Table 1 as following:

Table 1 Probabilities of survival benefit (increased percentage of patients alive at 5 years following D2) being greater than the specified target

\begin{tabular}{lcc}
\hline $\begin{array}{l}\text { Target survival } \\
\text { improvement }\end{array}$ & $\begin{array}{c}\text { Uninformative } \\
\text { prior }\end{array}$ & $\begin{array}{c}\text { Enthusiastic } \\
\text { prior }\end{array}$ \\
\hline $0 \%$ & 0.21 & 0.66 \\
$5 \%$ & 0.02 & 0.12 \\
$10 \%$ & 0.001 & 0.004 \\
\hline
\end{tabular}

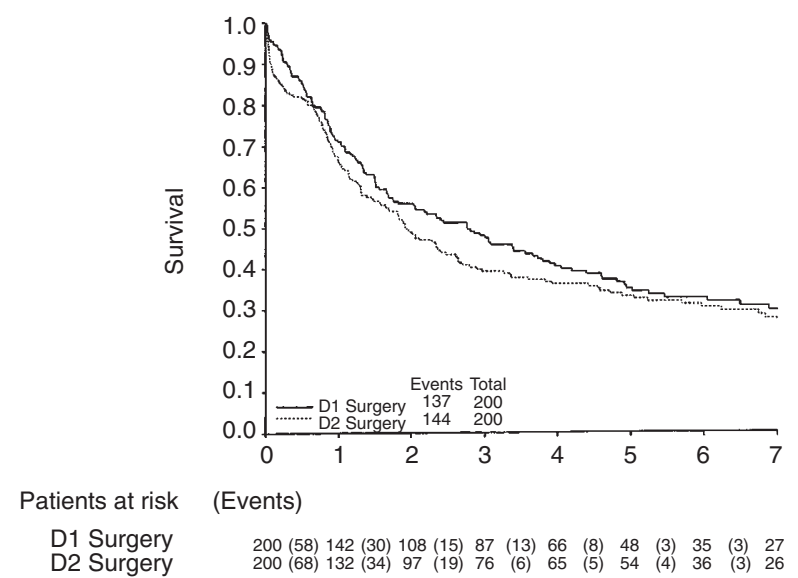

Figure 4 Survival in ST01

The 'uninformative prior' represents an open mind, with no prior opinion regarding the differences between D1 and D2. This corresponds more or less to conventional significance testing. This analysis of the ST01 results shows that there is very little chance of a $5 \%$ improvement, and a negligible chance of a $10 \%$ benefit. The 'enthusiastic prior' incorporates an adjustment based upon the optimistic beliefs expressed in the 1994 survey. In principle, even a small amount of confirmatory evidence from a clinical trial is likely to suffice to convince an enthusiast that D2 is superior, and substantial negative evidence would be necessary to dissuade an enthusiast; the degree of evidence that is necessary will depend upon the strength and range of the enthusiast's prior beliefs. The method of calculation is illustrated in Fayers et al (1997). Although an enthusiastic prior suffices to make it plausible that there is a benefit to D2 that is greater than zero (probability = 0.66 ), it remains unlikely that there could be a $5 \%$ increase in survival and it is highly improbable that the gain is as large as $10 \%$.

\section{DIsCussion}

Clinical trials are often carried out by enthusiasts who are convinced that a new treatment is effective. It calls for considerable perseverance to design, seek and obtain resources, launch and execute a multicentre randomized controlled trial. Many would only embark upon such a course if they believed that the new treatment potentially represents a major breakthrough. In view of this, it is perhaps not surprising that the trial steering committee started with opinions that were appreciably more optimistic than those of their colleagues. The initial expectations of this committee mellowed over time, and they became consistent with the more generally expressed opinions.

The opinions of the steering committee had changed substantially. Since the MRC Cancer Trials Office maintains extremely strict confidentiality of clinical trial data until patient accrual is completed, no hint of the results was known to anyone outside the office until the trial was closed. Thus any modification of opinions would be likely to be due to such influences as personal experience with D1 and D2 surgery, prevailing national and international opinion, publications in journals, and hearsay. Whilst we have 
confidence in the results of the second survey with respect to the steering committee members and some of the other surgeons, it is important to bear in mind that this survey was intentionally re-questioning the same surgeons that were surveyed 9 years previously. Reservations must be expressed as to how realistically the average expectations expressed in 1994 represent the prevailing consensus opinion. Some of those included in this later survey had retired from active work, and others were no longer practising in this area of surgery. Also, many surgeons active in gastric surgery in 1994 were explicitly excluded because they had not been surveyed in 1986; some of these would have been enthusiastic supporters for D2 surgery. Our sample was in no way a random sample of current UK gastric surgeons.

The value of our results is in terms of their implications for trial design. In particular, the results indicate that those involved in trial planning will often be more optimistic about treatment effects than their peers, and these opinions may well become modified - and arguably more realistic - as the time progresses. Hence a trial design committee may well aim to detect larger treatment effects than are perhaps realistic. This can lead to a smaller sample size than is necessary, with the attendant problems of low power to detect treatment benefits.

There is still some confusion over the operational definition of the alternative hypothesis in sample size calculations for clinical trials. Spiegelhalter and Freedman (1986) discussed the confusion between clinical demands and clinical expectations in this regard. They pointed out that both are necessary elements in sample-size determination but that the expectations should be the main guide to choosing the alternative hypothesis. They quantified clinical demands in terms of the range of equivalence. The upper limit of this range is the minimum improvement that is considered clinically important. Many investigators writing about sample-size determination have advised that the alternative hypothesis represents a clinically important difference. Some have specified that it be the smallest clinically important difference, that is, the upper limit of equivalence. This upper limit represents the smallest improvement of importance, and if there were sufficient power to detect this then there would be sufficient power to detect any important difference in the same direction. The downside to such a suggestion is that usually the sample size based on such a policy will be huge, and the policy would therefore tend to dissuade researchers from starting trials. In our particular trial the nonsteering committee clinicians' opinion of the expected benefit was actually quite close to the upper limit of equivalence, but it will not be so in all circumstances. For trials where there is a realistic expectation of an improvement much above the upper limit of equivalence the policy would demand a sample size far in excess of what is required. We therefore think it is preferable to base the alternative hypothesis on clinicians' opinion of the likely benefit, but taking trouble to obtain an opinion that is widely-based and not reliant on one or two enthusiasts.

For this trial a novel method of assessing clinicians' opinions was used, involving a detailed questionnaire answered by experienced gastric surgeons. The opinions expressed in the survey were consistent with the views of the trial planning committee, that the trial should aim to detect a change in 5-year survival from $20 \%$ (D1) to $33 \%$ (D2). This method proved invaluable for elucidating clinicians' opinions, and obtaining a general feel as to the difference of interest. Clinicians voiced the opinion that it made them think deeper about the forthcoming trial. ST01 was launched and recruited 400 patients as originally planned.
The initial baseline estimate of 5-year survival at 20\% was incorrect. This cannot be ascribed simply to improvement in surgical technique, since there are many other explanations that are equally or more likely. Not all patients are entered into clinical trials, and those recruited to ST01 may have been healthier than initially anticipated; in particular, patients could only be randomized if they were fit enough to be suitable for either operation. Medical care may have changed over the years. Cases may be diagnosed with earlier stage disease. More-rigorous staging procedures may have led to greater detection of advanced disease, with the subsequent exclusion of poor prognosis patients. It is interesting that at the second survey the trial steering committee surgeons correctly thought that the D1 survival rate of trial patients would be higher. This might partly be attributed to their own experience with patients who satisfied the eligibility criteria. In addition, although the trial results were confidential pending the completion of accrual, summary details of pre-randomization patient characteristics were available to the steering committee. These included tables showing the number of patients recruited by stage and extent of disease at presentation.

Over the 8 years that the trial was open to patient recruitment, many surgeons modified their views about D2 surgery. By 1994, most of those originally surveyed had come to believe that a $13 \%$ difference was too optimistic. The consensus opinion had become that $9-10 \%$ difference would be more realistic, and that this would still represent a sufficiently large difference to influence future surgical practice. Repeating the calculations for 32\% survival in D1 and $41.5 \%$ in D2 (a 9.5\% improvement), over 1000 patients would be required. This is more than double the number of patients in the ST01 trial, and would have required another 10 years recruitment. It was decided not to extend patient recruitment. This decision was partially based upon knowledge of a parallel trial that was being conducted in The Netherlands, and the two groups have agreed to carry out a joint analysis when the 10-year survival data of the two randomized trials becomes available.

\section{CONCLUSIONS}

Decisions about sample size are one of the most important aspects of clinical trial design. Funding bodies, ethical review committees and many medical journals all require explicit description of prestudy estimation of sample size and power. Many people recognize that it is rarely of any relevance to quote a precise estimate of the number of patients required, and it is customary to liberally round any sample size estimates upward. Despite this, even carefully designed trials may use sample sizes that, with hindsight, may be seen to be unrealistically small. We suggest that those designing trials should be circumspect about the optimism that may be expressed by trial planning committees. One possibility is to base sample size estimates upon a survey of clinical specialists. Maybe the views of those on the design committee should be excluded as being potentially biased.

For ST01, the survey of potential participants about their prior beliefs was being tried on an experimental basis and was not used to affect the study design. Since then, the method of surveying clinical opinion is one that the MRC Cancer Trials Office has been employing for an increasing number of trials, both surgical and non-surgical. We usually survey both intending participants and non-participants, to ensure broad coverage of opinions. It is a method that we advocate for wider use. In our experience, clinicians are actively interested in participating in such exercises, and 
the information collected is very helpful towards ensuring that the trial is designed as realistically as possible. This approach, when used for trial design, enables the opinions of those outside the planning committee to be incorporated into the design process.

An important aspect of the approach is the recognition of a 'range of equivalence'. Although this may at first sight be thought to be an unnecessary complication, our experience confirms that this concept reflects the way that clinicians think about treatment benefits. Many clinicians would find it more difficult to answer the apparently simpler question about the level of treatment benefit is important and worthwhile.

We recommend that both the expected changes and the range of equivalence should be assessed in a survey when designing a clinical trial. It is important to confirm that the anticipated treatment effect would be large enough to cause a change of practice in a reasonable proportion of clinicians, and that it would therefore be regarded as clinically relevant. Both the expected treatment effect and the range of equivalence should be considered for sample-size estimation.

In addition to their implication for sample size estimation, the elicitation of clinicians' prior beliefs can also be incorporated in a Bayesian approach to analysis of clinical trials. This allows interpretation of the results to be influenced by the optimistic - or, possibly, sceptical - opinions of the medical community.

\section{CONTRIBUTORS}

Alfred Cuschieri was the chairman of the ST01 planning and steering committees, from 1985 until 1997, and John Fielding and John Craven were the two other surgical members. Peter Fayers was study statistician from 1989 to 1997 , and was responsible for the second survey. The initial 1986 survey was the idea of Laurence Freedman, who was also responsible for carrying it out. Barbara Uscinska took on the difficult task of tracing the surgeons who had participated in the initial survey, and persuading those still alive to repeat the exercise in 1995. All of these named persons were involved in the writing of this report.

\section{ACKNOWLEDGEMENTS}

In addition to those surgeons on the writing committee $(\mathrm{AC}, \mathrm{JF}$, $\mathrm{JC})$, the following were involved in the two surveys and we acknowledge their help: J Banciewicz, A I M Cook*, W M Cooke*, M J Cooper*, N J Dorricott*, G H Dunstone†, D J Ellis*, A W Hall*, R I Hall†, M J Gough*, M J McMahon*, M C Pietroni*, A Pollock*, J G Roberts $\uparrow$, G R Sagor*, R D Stedeford*, C Stoddard*, T V Taylor*, J G Temple†, D E F Tweedle*, C M White*, H S Winsey†, R A B Wood* (*participant in 1986 and 1995 surveys; $\uparrow$ participant in 1986 only).

\section{REFERENCES}

Cuschieri A, Fayers PM, Fielding JWL, Craven JL, Bancewicz J, Joypaul V and Cook P (1996) Postoperative morbidity and mortality after D1 and D2 resections for gastric cancer - results of the MRC randomised controlled trial. Lancet 347: 995-999

Cuschieri A, Weeden S, Fielding J, Banciewicz J, Craven J, Joypaul V, Sydes M, Fayers P, for the Surgical Cooperative Group (1999) Patient survival after D1 and D2 resections for gastric cancer: long-term results of the MRC randomised surgical trial. Br J Cancer 79: 1522-1530

Fayers PM and Machin D (1995) Sample size: how many patients are necessary? Br J Cancer 72: 1-9

Fayers PM, Ashby D and Parmar MKB (1997) Tutorial in biostatistics: Bayesian data monitoring in clinical trials. Stat Med 16: 1413-1430

Machin D, Campbell MJ, Fayers PM and Pinol A (1997) Sample Size Tables for Clinical Studies, 2nd edn. Blackwell Science: Oxford

Maruyama K, Okabayashi K and Kinoshita T (1987) Progress in gastric cancer surgery and its limits of radicality. World J Surg 11: 418-426

Parmar MKB, Spiegelhalter DJ and Freedman LS (1994) The CHART trials: Bayesian design and monitoring in practice. Stat Med 13: 1297-1312

Spiegelhalter DJ and Freedman LS (1986) a predictive approach to selecting the size of a clinical trial, based upon subjective clinical opinion. Stat Med 5: 1-13. 